\title{
Interstellar Processes Leading to Molecular Deuterium Enrichment and Their Detection
}

\author{
Scott A. Sandford
}

NASA-Ames Research Center, Mail Stop 245-6, Moffett Field, CA 94035-1000 USA

*Author to whom correspondence should be addressed: Dr. Scott A. Sandford

NASA-Ames Research Center

Mail Stop 245-6

Moffett Field, CA 94035

Phone: 650-604-6849

Fax: 650-604-6779

Email: ssandford@mail.arc.nasa.gov

Submited to Planetary and Space Science, October 10, 2001

26 Pages (not including Figures)

6 Figures

1 Table

Key Words: deuterium e:richment; interstellar medium; interstellar chemistry; polycyclic aromatic hydrocarbons; isotopic anomalies; ultraviolet photolysis; organics 


\begin{abstract}
Large deuterium (D) enrichments in meteoritic materials indicate that interstellar organic materials survived incorporation into parent bodies within the forming Solar System. These enrichments are likely due to one or more of four distinct astrochemical processes. These are (i) low temperature gas phase ion-molecule reactions, (ii) low temperature gas-grain reactions, (iii) gas phase unimolecular photodissociation, and (iv) ultraviolet photolysis in Denriched ice mantles. Each of these processes should be associated with molecular carriers having distinct regiochemical signatures (D placement on the product molecules, correlation with specific chemical functionalities, etc.). These processes are reviewed and specific spectroscopic signatures for the detection of these processes in space are identified and described.
\end{abstract}

\title{
1. INTRODUCTION
}

Isotopic measurements, primarily in the form of deuterium (D) enrichments, provide definitive evidence for presolar interstellar materials within meteoritic samples (Robert and Epstein, 1982; Yang and Epstein, 1983; Kerridge and Chang, 1985; Kerridge et al., 1987). These enrichments are seen both in bulk meteoritic materials (Zinner, 1988), in meteoritic subfractions, and in specific classes of molecular species, such as amino and carboxylic acids (Epstein et al., 1987; Pizzarello et al., 1991; Krishnamurthy et al., 1992; Zinner, 1997).

The traditional explanation of these meteoritic $D$ excesses has been to invoke the presence of materials made in the ISM by low temperature gas phase ion-molecule reactions (Geiss and Reeves, 1981; Dalgarno and Lepp, 1984). The D/H ratios of simple interstellar molecules amenable to measurement using radio spectral techniques are generally higher than those of Solar System materials (Robert et al., 2000). However, comparisons of meteoritic and 
interstellar $\mathrm{D} / \mathrm{H}$ ratios are obscured by several effects. First, $\mathrm{D}$ enrichments in interstellar molecules have been detected in only a few simple species, molecules that are not the principle carriers of D in Solar System materials. Second, some of the observed interstellar D enrichments reside on labile molecular sites that would be expected to exchange to some degree with more isotopically normal material during incorporation into a warm protosolar nebula, parent body processing, delivery, recovery, and analysis. Finally, ion-molecule reactions represent only one of four different processes: that can produce strong D-H fractionation in the ISM (Sandford et al., 2001).

The four chemical processes that can lead to $D$ enrichment are briefly reviewed in $\S 2$. These processes are expected to manifest in ways that are amenable to telescopic observation. Section 3 provides a disclission of the infrared spectral signatures that should be associated with D enrichment in the ISM and that may serve to delineate the contributions of the different chemical processes to $D$ enrichment. Finally, $\$ 4$ provides a very brief discussion of how isotopic investigations of meteoritic organics may help establish the relative contributions of these interstellar processes to the $\mathrm{D}$ enrichments found in solar system materials.

\section{INTERSTELLAR CHEMICAL PROCESSES FOR DEUTERIUM ENRICHMENT}

Interstellar molecule:; including PAHs, can become D-enriched through the processes of gas phase ion-molecule re actions at low temperatures (Dalgarno and Lepp 1984; Tielens, 1997). gas-grain reactions (Tielens, 1983, 1992, 1997), unimolecular photodissociation (Allamandola et al., 1987, 1989), and radiation processing of D-enriched ice grains (Sandford et al., 2000). Each of these processes manifests itself in the production of D-enriched molecules with distinctive regiochemical signatures (Sandford et al., 2001). These differences are summarized in Table 1. 
For the purpose of inter-process comparisons, the chemistry of polycyclic aromatic hydrocarbons (PAHs) will be highlighted since PAHs are abundant and widespread in the universe (Puget and Leger, 1989; Roelfsema et al., 1996; Allamandola et al., 1989, 1999), and PAHs are extremely stable molecules that represent one of the few classes of materials that can survive in all the interstellar environments associated with the four chemical processes listed above. In addition, PAHs and related aromatic materials are common in primitive meteorites (Cronin and Chang, 1993; Gardinier et al., 2000; Cody et al., 2001; and references therein) and interplanetary dust particles (IDPs) (Allamandola et al., 1987; Clemett et al., 1993), both of which contain significant carriers of D (Kerridge and Chang, 1985; McKeegan et al., 1985; Keller et al., 2000; Messenger, 2000).

For a more detailed description of these processes, as well as a discussion about how these processes are expected to effect C, N, and O isotopic ratios, see Sandford et al. (2001) and references therein.

\section{Low Temperature Gas Phase Ion-Molecule Reactions}

Deuterium fractionation by ion-molecule reactions occurs only at the very low temperatures characteristic of dense interstellar clouds $(\mathrm{T}<50 \mathrm{~K})$. At such temperatures, chemical reactions between most neutral gas phase species will not proceed at any appreciable rate, but exothermic ion-molecule reactions can occur even at very low temperatures (cf. Geiss and Reeves, 1981; Herbst, 1987; Tielens, 1997). Thus, most of the gas phase chemistry occurring in dense clouds is the result of ion-molecule reactions and exoergic reactions involving radicals.

Such reactions are expected to produce mass fractionation at low temperatures due to the different zero-point energies of $H$ and $D, D$ having a lower energy and the potential to form slightly stronger chemical bonds (Dalgarno and Lepp, 1984; Tielens, 1997; Millar et al., 2000). 
At high temperatures fractionation is a minor effect, but as the gas drops below about $70 \mathrm{~K}$, fractionation becomes increasingly more significant (e.g. Millar et al., 1989). This process can proceed with most $\mathrm{H}$-bearing gas phase molecules. This includes gas phase PAHs (Tielens, 1997, Figure 1 shows the case for the PAH pyrene). This process is expected to initially preferentially deuterate the larger PAHs in dense clouds since they have more $\mathrm{H}$ atoms, but ultimately all PAHs in the gas phase should show increasing D/H ratios as they approach steadystate equilibrium with $\mathrm{H}$ in the gas phase. Since $\mathrm{PAHs}$ are considerably more stable and more abundant in the ISM than many of the smaller species that show $\mathrm{D}$ enrichments $\left(\mathrm{PAH} / \mathrm{H}_{2}-10^{-6}\right.$ compared to $\mathrm{HCN} / \mathrm{H}_{2} \sim 10^{-8}$ ), and PAHs carry more $\mathrm{H}$ atoms per molecule than simpler molecules, PAHs are likely to represent the largest reservoir of materials D-enriched by ionmolecule reactions.

Observational evidence that $D$ fractionation occurs in the ISM via gas phase ion-molecule reactions comes from radio telescopic studies that have demonstrated that several simple molecules are often highly D-enriched in dense interstellar clouds (Jefferts et al., 1973; Hollis et al., 1976; Mauersberger et al., 1988). Deuterium enrichments in interstellar PAHs have not yet been detected (or carefully searched for).

\section{Low Temperature Gas-Grain Reactions}

Ion-molecule reactions can only occur in the gas phase. However, at the temperatures typical of dense clouds $(\mathrm{T}<50 \mathrm{~K}$ ) most volatile species are condensed out of the gas phase into icy grain mantles (Sandford and Allamandola 1993), as evidenced by the large quantities of mixedmolecular ices (Sandford, 1996) and gas phase depletions (Mauersberger et al., 1992) seen in dense clouds. The compssition of these ices does not simply reflect gas phase abundances, however, since additional species are formed when condensing species participate in gas-grain 
reactions on the grain surfaces (Tielens and Hagen, 1982; d'Hendecourt et al., 1985; Brown and Charnley, 1990; Hasegawa et al., 1992). The compositions of these new species are strongly dependent on the local $\mathrm{H}_{2} \mathrm{H}_{2}$ ratio. In environments where $\mathrm{H} / \mathrm{H}_{2}$ is large, surface reactions with $\mathrm{H}$ atoms dominate and species like $\mathrm{CH}_{4}, \mathrm{NH}_{3}$, and $\mathrm{H}_{2} \mathrm{O}$ are produced. If $\mathrm{H} / \mathrm{H}_{2}$ is small, reactive species such as $\mathrm{O}$ and $\mathrm{N}$ can react with one another to form $\mathrm{O}_{2}$ and $\mathrm{N}_{2}$.

Gas-grain chemistry should produce large $D$ enrichments in grain mantles where reduction is occurring (Tielens, 1992, 1997). In this case, the role of the zero-point energy difference between $H$ and D counterparts is limited (Tielens, 1983). Instead, D enrichment results primarily from the high atomic $D$ fractionation of the accreting gas. Both the atomic $H$ and $D$ accreted onto grain surfaces can tunnel through activation barriers and hydrogenate species that are not fully reduced. Models indicate that such a process could yield mantle $\mathrm{D} / \mathrm{H}$ ratios as high as 0.1 for simple molecules like $\mathrm{H}_{2} \mathrm{O}$ and $\mathrm{CH}_{3} \mathrm{OH}$ (Tielens, 1983, 1992, 1997; Charnley et al., 1997). Values in this range have been reported for $\mathrm{HDO} / \mathrm{H}_{2} \mathrm{O}$ in interstellar ices (Teixeira et al., 1999). Since the grain mantles in dense clouds represent a much larger fraction of the total reservoir of material than does material in the gas phase $\left(\mathrm{H}_{2}\right.$ excepted), grain surface processes are probably far more important for the total $D$ fractionation in dense clouds than ion-molecule reactions.

Naturally, D fractionation during gas-grain reactions should only proceed efficiently for molecules that can be hydrogenated. Fully reduced species will not accept additional $D$ in this fashion and simple exchange is unlikely under these low temperature conditions. In the case of PAHs, such reduction would result in the production of $H_{n}-$ PAHs, aromatic species in which excess $\mathrm{H}$ atoms convert some of the aromatic rings to aliphatic rings (Bemstein et al., 1996, Figure 2). There is currently no laboratory evidence suggesting that PAHs can become further reduced in this manner through simple $\mathrm{H}$-atom exposure on grain surfaces. Thus, while gas- 
grain reactions may dominate the mass distribution of $D$ in dense clouds, it may leave PAHs largely unaffected. However, to the extent that this process does occur to PAHs, any D enrichments generated would be expected to correlate with the presence of $H_{n}-P A H s$, increasing $\mathrm{H} / \mathrm{C}$ ratios, and the presence of aliphatic structures (Table 1).

\section{Unimolecular Photodissociation Reactions}

In contrast to ion-molecule and gas-grain reaction processes, D-enrichment by unimolecular photodissociation does not require low temperatures and is expected to be restricted solely to PAHs (Allamandola et al., 1987, 1989; Tielens, 1997). Because of their multiple C bonding and $\pi$ electrons, PAHs are fáirly resistant to photolytic destruction and can survive in environments where other species would be quickly fragmented. While relatively immune to complete photodestruction by interstellar UV photons, individual PAHs may still absorb UV photons that contain more energy than they can vibrationally accommodate. In such cases, a bond or bonds will break, the most likely being a peripheral $\mathrm{C}-\mathrm{H}$ bond followed by loss of the $\mathrm{H}$ atom. Because of the zero-point energy difference of the C-D and C-H bonds, the rate of D loss from interstellar PAHs should be lower than that of $\mathrm{H}$ (Figure 3 ). The resulting PAH radical will subsequently react with ambient H/D atoms, and repeated processing should gradually enrich interstellar PAHs in D relative to the ambient gas. Steady state equilibrium is expected to be reached in photodissociation regions and, at least for small PAHs, in dense clouds. At equilibrium, the fraction of peripheral $\mathrm{D}$ relative to $\mathrm{H}$ will be 3 times the local gas phase ratio of $\mathrm{D}$ to $\mathrm{H}\left(10^{-5}\right.$ to $10^{-3}$ ) (Allamandola et al , 1989; Tielens, 1997). Thus, in these interstellar environments, many PAHs having less than $.25 \mathrm{C}$ atoms should have peripheral $\mathrm{D}$ atoms.

This process is unique in two ways. First, low ambient temperatures are not required to produce fractionations $t y$ this process. Second, the extent of the D-enrichment depends on the 
size of the PAH. D enrichment is expected to be most significant for PAHs in the size range from $\mathrm{C}_{10} \mathrm{H}_{8}$ (naphthalene) to $\mathrm{C}_{42} \mathrm{H}_{18}$ (hexabenzocoronene). Enrichment in PAHs having more than $\sim 40 \mathrm{C}$ atoms is not expected because these larger PAHs have larger numbers of vibrational modes, and can therefore accommodate the maximum energy of typical UV photons without photolytic bond cleavage occurring (Figure 3 ).

\section{Ultraviolet Photolysis Reactions in Deuterium-enriched Ice Mantles}

Most of the volatile species in dense clouds reside in icy grain mantles. The dominant species in these ices is usually $\mathrm{H}_{2} \mathrm{O}$, but they also contain $\mathrm{CO}, \mathrm{CO}_{2}, \mathrm{CH}_{3} \mathrm{OH}, \mathrm{NH}_{3}, \mathrm{H}_{2} \mathrm{CO}_{,} \mathrm{CH}_{4}$, and a number of other simple species in abundances greater than $1 \%$ of $\mathrm{H}_{2} \mathrm{O}$ (see Sandford, 1996; Whittet et al., 1996). Many of these species should be significantly enriched in D, either by enrichment in the gas phase by ion-molecule reactions followed by condensation onto the grain mantle, or via gas-grain reactions occurring on the mantle surface. Once in the ice, these molecules can serve as a D-enriched reservoir for the synthesis of other enriched species.

One would normally expect few chemical reactions to occur within these ice mantles because of their low temperatures. However, interstellar ices in dense clouds can be further modified by energetic processing by ambient UV radiation and cosmic rays (Norman and Silk, 1980; Prasad and Tarafdar, 1983). When these ices are irradiated and warmed, bonds are broken, molecules are destroyed, and new molecules, radicals, and ions are formed. Thus, irradiation and thermal processing of ices made of simpler (D-enriched) molecules is expected to form a host of more complex species in dense interstellar clouds (Moore et al., 1983: Agarwal et al., 1985; Allamandola et al., 1988; Bernstein et al., 1995; Dworkin et al., 2001). Infrared spectra of the ices in dense clouds provide evidence for this process (Tegler et al., 1993; Pendleton et al., 1999; Bernstein et al., 2000). Furthermore, laboratory studies of mixed-molecular ices in which some of the original 
species are D-enriched have shown D incorporation into many of the reaction products (Bernstein et al., 1994, 1995, 2000; Sandford et al., 2000). Unlike the previously described processes, the effect is not to produce a fractionation of $\mathrm{H}$ from $\mathrm{D}$, but instead to propagate previous fractionations into new molecular species, i.e., the "global" $\mathrm{D} / \mathrm{H}$ ratio of the ice mantle remains fixed, but the $\mathrm{D} / \mathrm{H}$ ratios in individual molecular families may be redistributed into other species.

The presence of PAHs in these environments has been demonstrated by detection of their infrared vibrational bands in absorption in the spectra of stars embedded within dense clouds (Sellgren et al., 1995; Brooke et al., 1996, 1999; Chiar et al., 2000; Bregman et al., 2000). Once condensed into interstellar ice mantles, PAHs will be subjected to the same radiation processing as the other ice components. Laboratory irradiation simulations have shown that under interstellar conditions PAHs undergo both oxidation and reduction reactions that affect their edge structures (Bernstein et al , 1999, 2001). Oxidation results in the production of aromatic alcohols, ketones, and eth $2 r s$, while reduction leads to the formation of $\mathrm{H}_{n}$-PAHs. Laboratory studies of PAHs in D-enriched $\mathrm{H}_{2} \mathrm{O}$ ices have shown that UV photolysis results in rapid D enrichment of the PAHs through aromatic $\mathrm{D} \rightarrow \mathrm{H}$ exchange, $\mathrm{D}$-atom addition, and exchange through keto-enol tautomerism. Each of these processes produce different compounds with characteristic deuteration patterns and D labilities (Sandford et al., 2000): $\mathrm{D} \rightarrow \mathrm{H}$ exchange reactions result in enriche 1 PAHs in which the $D$ resides in relatively non-labile locations (Figure 4a), D-atom addition reactions result in enriched $H_{n}-P A H s$ in which the D resides in relatively non-labile aliphatic rings attached to aromatic rings (Figure $4 \mathrm{~b}$ ), and enrichment via keto-enol tautomeric exch ange results in the $\mathrm{D}$ being located exclusively on rings that contain oxygen atoms in sites that are relatively labile (Figure $4 c$ ). 
These deuterium enrichments are expected to occur largely independent of PAH molecular size, but should show specific regiochemical behaviors. In addition, since this same irradiation process produces both reduced PAHs $\left(\mathrm{H}_{\mathrm{n}}-\mathrm{PAHs}\right.$ ) and oxidized PAHs (aromatic alcohols, ketones, and ethers) (Bernstein et al., 1999), D enrichments produced by this process would be expected to correlate with the presence of these other species (Table 1).

\section{SEARCHING FOR D ENRICHMENTS IN INTERSTELLAR MOLECULES}

\section{The Difficulties Associated with Detecting Deuterated Molecules in Space}

The chief difficulty of studying deuterated molecules in space is the low intrinsic abundance of $D$. The current cosmic $D / H$ ratio is on the order of $1 \times 10^{-5}$. In environments where $D$ fractionation is occurring this ratio will be larger in select molecules, but even under the most efficient fractionation this ratio is generally not expected to exceed a value of 0.01 , or perhaps 0.1 . Thus, studies of the fractionation of $D$ within specific molecular species requires the measurement of both the protonated molecule and its deuterated variant, which will be at least an order of magnitude less abundant, but more likely less abundant by factors of $10^{2}-10^{4}$. This places stringent requirements on the required sensitivities and signal-to-noise ratios of such studies.

The best technique for the detection of D-enriched molecules in space is the spectroscopic measurement of bands whose positions are shifted by the presence of $D$, i.e., molecular rotational and vibrational transitions. Past studies have concentrated almost exclusively on the use of spectroscopy at the radio frequencies where many of the rotational lines of molecules with significant dipole moments are found. The greater relative mass of the $D$ atom in deuterated molecules can cause significant displacement in the rotational band positions and allows for the quantitative determination of $\mathrm{D} / \mathrm{H}$ ratios through the comparison of the line strengths of gas phase molecules and their deuterated counterparts. For example, radio observations have lead to the 
detection of large D enrichments in simple molecular systems like DCN/HCN (Jefferts et al., 1973), $\mathrm{DCO}^{+} / \mathrm{HCO}^{+}$(Hollis et al., 1976), and $\mathrm{CH}_{3} \mathrm{OD} / \mathrm{CH}_{3} \mathrm{OH}$ (Mauersberger et al. 1988). Such observations are well suited to the study of D enrichments in small gas phase molecules, particularly as they serve as probes to the fractionation processes associated with ion-molecule reactions.

Enrichment studies at radio wavelengths have some limitations, the most significant being that the technique is restricted to molecules in the gas phase. Three of the four fractionation processes operate only at very low temperatures, where most molecular species will be condensed out of the gas phase and into icy grain mantles. In addition, even in the gas phase, molecules will be undetectable if they have very small dipole moments or are very large. This precludes the use of these techniques for the study of D enrichment in gas phase PAHs and the unimolecular photodissociation enrichrient process. Thus, while radio observations provide an excellent means of probing the $\mathrm{D} / \mathrm{H}$ ratios of certain specific molecular systems, they cannot be used to study most of the material that is likely to be carrying excess. Thus, radio observations alone cannot provide an understanding of the relative importance of the four different fractionation processes.

If the bulk of the cariers of $D$ in space are to be identified and understood, it will be necessary to probe the solid components of the interstellar medium. The chief means of doing this is also spectroscopic, although at infrared (IR) wavelengths where the principal vibrational transitions of molecules le. IR spectroscopy has the advantage of not being restricted solely to gas phase species. Unforcunately, relatively little work has been done on the measurement of D enrichments at these wavelengths. The recent IR detection of greatly elevated $\mathrm{HDO} / \mathrm{H}_{2} \mathrm{O}$ ratios in interstellar ices (Teixeira et al., 1999) suggests, however, that infrared studies could play a large role in the future study of interstellar D fractionation chemistry. 
One significant obstacle to the infrared measurement of D-enriched molecules is the Earth's atmosphere. The principle means of measuring $D$ in molecules in the infrared is through the detection of vibrational stretching or bending modes involving C-D, O-D, and N-D bonds. Unfortunately, these are obscured by the asymmetric stretching and bending modes of telluric $\mathrm{CO}_{2}$ (Figures 5 and 6). Thus, spaceborne platforms are required for such studies (the work by Teixeira et al. (1999) was done using the ISO space telescope).

\section{Using Infrared Spectra to Study Interstellar Deuterium Fractionation Processes}

Key to the IR spectral study of interstellar D fractionation is the ability to measure very high signal-to-noise spectra of appropriate interstellar environments at the wavelengths characteristic of $\mathrm{C}-\mathrm{H}, \mathrm{O}-\mathrm{H}$, and $\mathrm{N}-\mathrm{H}$, and their corresponding C-D, O-D, and N-D, stretching and bending mode vibrations. As an example, Figures $5 \mathrm{a}$ and $5 \mathrm{~b}$ shows the infrared $\mathrm{C}-\mathrm{H}$ stretching and bending mode bands, respectively, of four different PAHs and their perdeuterated counterparts. Both the $\mathrm{C}-\mathrm{H}$ stretching and bending modes are significantly shifted to longer wavelengths in the deuterated variants; the aromatic $\mathrm{C}-\mathrm{H}$ stretch shifts from near $3.3 \mu \mathrm{m}$ to around $4.4 \mu \mathrm{m}$ and the various $\mathrm{C}-\mathrm{H}$ bending modes shift from the $11-15 \mu \mathrm{m}$ region to the $15-18 \mu \mathrm{m}$ region.

Thus, in principle, the D/H ratios in PAHs (as one example) could be measured by comparing the relative strengths of the corresponding $\mathrm{C}-\mathrm{H}$ and $\mathrm{C}-\mathrm{D}$ modes. As noted earlier, this will require a spaceborne telescope and very high quality spectra. Figure 6 shows the spectrum of a mixture of PAHs having an overall $\mathrm{D} / \mathrm{H}$ ratio of 0.05 , i.e., an enrichment comparable to the biggest that might be expected in favorable environments space. It is immediately apparent that the $\mathrm{C}$-D modes of $\mathrm{D}$-enriched molecules will only be detectable in spectra with high signal-tonoise. 
With sufficient sensitivity, spectra coverage, and spectral resolution, there are several tests that could help constrain the relative importance of the various interstellar $\mathrm{D}$ fractionation mechanisms. In the case of $D$ enrichment via ion-molecule reactions, it would be useful if the presence of D-containing $\mathrm{PAH}^{+}$ions could be verified in the gas phase in dense interstellar clouds. In principle, it m 1y be possible to determine PAD/PAH ratios at infrared frequencies using the relative strengtt s of the aromatic $\mathrm{C}-\mathrm{H}$ and $\mathrm{C}-\mathrm{D}$ stretching emission bands near 3050 and $2270 \mathrm{~cm}^{-1}$, respectively (Hudgins et al., 1994). However, the low expected gas phase abundances of PAHs in such cold environments will make this very difficult. In addition, PAHs are most easily observed in the gas phase when they are excited by UV photons and then radiatively cool by emitting a cascade of infrared photons. At the relatively low UV fluxes present in typical dense cloud environment:; this excitation mechanism is strongly curtailed. There may be more hope of measuring the relative strengths of the $\mathrm{C}-\mathrm{H}$ and $\mathrm{C}-\mathrm{D}$ aromatic stretching bands in absorption, but in this case the bands would presumably be dominated by PAHs frozen onto grains and it would be difficult to assign any observed fractionation specifically to ion-molecule reactions, as opposed to cther processes.

The same UV excitai ion that makes gas phase PAHs observable via infrared emission should also produce $\mathrm{D}$ en-ichment by unimolecular photodissociation teactions, with the largest enrichments occur in PAHs smaller than $\sim \mathrm{C}_{50}$. This is in contrast to ion-molecule reactions, which should tend to preferentially enrich larger PAHs. The challenge will be to use the infrared C-D and C-H stretching emission bands near 4.40 and $3.28 \mu \mathrm{m}$, respectively, to measure the PAD/PAH ratio in a variety of interstellar environments that sample different UV radiation fields and astrochemical evolutionary histories. If $D$-enrichment by unimolecular photodissociation is occurring. PAD/PAH ratiss should be very low in the outflows from carbon stars, where $\mathrm{D}$ is 
largely absent, having been consumed in the star. PAD/PAH ratios should be much larger in diffuse interstellar 'cirrus' clouds, $\mathrm{H}$ II regions, and reflection nebulae, environments where PAHs have longer UV exposures in environments with higher gas phase $\mathrm{D} / \mathrm{H}$ ratios.

\section{The Dependence of Deuterium Enrichment on Environment}

Previous sections have described how different chemical processes are expected to produce different $\mathrm{D} / \mathrm{H}$ ratios among co-spatially located molecular species. However, these multiple chemical routes to $\mathrm{D}$ fractionation have different dependencies on various environmental parameters (temperature, $\mathrm{H}_{2} \mathrm{H}_{2}$ ratio, $\mathrm{UV}$ and cosmic ray flux, etc.), and it is expected that the $\mathrm{D} / \mathrm{H}$ ratios of a given molecular species should vary between environments. Since many environments, dense molecular clouds for example, can support more that one D fractionation process, the dependence of fractionation on environmental conditions could be difficult to determine.

In general, significant $D$ fractionations are not expected in molecular species found in the outflows of aging stars (AGB stars, novae, etc.). This is largely because most of the D original to the star would have been destroyed and little $D$ would be expected in their outflows. In addition, the materials in outflows are quite young and therefore will have had time to participate in relatively little chemical and isotopic exchange. For this reason, it is not anticipated that molecular species in C-rich planetary nebulae, where PAHs are very abundant, will show any appreciable level of $\mathrm{D}$.

It should be easier to detect deuterated species, including PAHs, in dense clouds. This is both because all four of the possible $D$ enrichment mechanisms can operate in these environments and because the contents of these clouds represent materials that have been exposed to much longer periods of chemical evolution during which D excesses could have accumulated. 
However, the possible sirnultaneous action of all four processes will also make it more difficult to delineate which processes are responsible for any detected enrichments. A clear understanding will require the correlation of $\mathrm{D}$ enrichments with environmental conditions spanning a wide range of temperatures, de 1sities, dynamics, radiation fluxes, etc.

\section{SOME IMPLICATIONS FOR METEORITIC ORGANICS}

The relative importance of these various astrochemical processes can, in principle, be garnered from the identities, abundances, and regiochemistry of the carriers of excess $D$ in meteorites and DPS (Sandford et al., 2001). Unfortunately, there is currently little information about the nature of the $D$ carriers within the organic fraction of meteorites, either in terms of their distribution among the various molecular species present or the siting of the $\mathrm{D}$ within these species.

Deuterium enrichmer ts have been identified in a number of specific classes of molecules in meteorites, including amiıı and carboxylic acids (Epstein et al., 1987; Pizzarello et al., 1991; Krishnamurthy et al., 199?.) and aromatic hydrocarbons (cf. Robert and Epstein, 1982; Kerridge et al. 1987; Krishnamurthy' et al., 1992). Aromatic hydrocarbons and related materials are the main carrier of carbon in primitive meteorites (cf. Cronin et al., 1987; De Vries et al., 1993; Gardinier et al., 2000; Coc.y et al., 2001) and IDPs (Allamandola et al., 1987; Clemett et a1., 1993 ) and are therefore likely to be the main carriers of the D excesses as well (Kerridge et al. 1987).

On the basis of currer t knowledge, it is not possible to exclude potential contributions to the inventory of excess meteo itic $D$ from any of the four processes outlined above. For example, the report by Aléon et al. (2000) that the value of $\delta \mathrm{D}$ increases with increased C/H in IDPs, with most enrichments occurring when $\mathrm{C} / \mathrm{H}>1$ is qualitatively consistent with a fractionation of the $\mathrm{D}$ 
enrichment occurring in PAHs via ion-molecule reactions. However, the observation that the D/H ratio in acid insoluble phases drops slightly as the $\mathrm{C} / \mathrm{H}$ ratio increases from 2.3 to 3.0 (Robert and Epstein, 1982) is also qualitatively consistent with enrichment by unimolecular photodissociation (Allamandola et al., 1987). The suggestion that the D in IDPs may be carried in C-rich materials that contain aldehydes (Keller et al., 2000) is consistent with the photoenrichment of PAHs frozen in D-rich ices. The lack of correlation of $\mathrm{D}$ enrichment with $\mathrm{C}$ and $\mathrm{O}$ isotopic ratios and the poorly defined relationship between $\mathrm{D}$ enrichments and ${ }^{15} \mathrm{~N}$ enrichments are also compatible with these processes (Sandford et al., 2001).

Specific tests designed to address these meteoritic issues have been suggested (Sandford et al., 2001), and the capabilities needed to make these tests are fast becoming available. It can be anticipated that a great deal will be learned about the carriers of $D$ in meteorites, and the chemical processes whereby they formed, in the next few years.

\section{CONCLUSIONS}

There are at least four interstellar astrochemical processes that can produce significant $D$ fractionation in their products: gas phase ion-molecule reactions, low temperature gas-grain reactions, gas phase unimolecular photodissociation, and ultraviolet photolysis in D-enriched ice mantles. In the case of PAHs, these processes are associated with distinct regiochemical signatures. Gas phase ion-molecule reactions initially preferentially deuterate the larger PAHs in dense clouds, but if sufficient time is available, all PAHs in the gas phase in should reach equilibrium with the gas phase. Thus, the signature of this process would be fully aromatic PAHs whose D/H ratios are uniform across the PAH population or that increase with increasing PAH size. Gas-grain reactions produce D fractionations in dense clouds manifested primarily in simple hydrogenated molecules. PAHs probably cannot be enriched in D in this manner, but if 
they are, $\mathrm{D}$ enrichments would be found in $\mathrm{H}_{\mathrm{n}}$-PAHs, i.e., hydrogenated PAHs that contain cyclic aromatic and aliphatic rings. Enrichment via gas phase unimolecular photodissociation reactions should produce a fractionation pattern in which PAHs below the $\mathrm{C}_{50}$ size range show the largest enrichments. Finally, photochemistry of ices containing pre-existing enrichments may preserve the fractionations generated by other mechanisms in new forms that can survive incorporation into forming stellar systems. Deuterium enrichments produced in aromatic species by this process will be largely independent of PAH size and should show specific regiochemical behaviors like correlatiors between $\delta \mathrm{D}$ and the presence of both reduced PAHs $\left(\mathrm{H}_{\mathrm{n}}-\mathrm{PAHs}\right)$ and oxidized PAHs (aromatic alcohols, ketones, and ethers).

These signatures that can be searched for spectroscopically in the interstellar environments where these processes are operant, as well as in the molecular carriers of the excess $D$ found in the organic fractions of primitive meteorites. Deuterium-enriched molecules in space can be detected in through their spectral signatures, both through rotational transitions (at radio wavelengths) and throughı vibrational transitions (at infrared wavelengths). Since they are not restricted to gas phase species, infrared measurements are more likely to provide information about the distribution of the bulk of D-enriched species. Deuterated species are most easily identified in the infrared through their characteristic X-D stretching and bending mode vibrations, which fall in the spectral regions around 4.4 and $16.5 \mu \mathrm{m}$, respectively.

Acknowledgements - This work was supported by NASA grants 344-37-44-01 (Origins of Solar Systems), 344-38-12-04 (Exobiology), and 344-50-92-02 (Astrobiology). The author grateful acknowledges useful disc ıssions with M. P. Bernstein and J. P. Dworkin. 


\section{REFERENCES}

Agarwal, V.K., Schutte, W., Greenberg, J.M., Ferris, J.P., Briggs, R., Connor, S., Van De Bult, C.P.E.M., Baas, F., 1985. Photochemical reactions in interstellar grains. Photolysis of CO, $\mathrm{NH}_{3}$ and $\mathrm{H}_{2} \mathrm{O}$. Origins of Life 16, 21-40.

Aléon, J., Engrand, C., Robert, F., Chaussidon, M., 2000. Isotopic study of the potential carriers of deuterium excesses in interplanetary dust particles. Meteoritics and Planetary Science 35, A19-A20.

Allamandola, L.J., Sandford, S.A., Wopenka, B., 1987. Interstellar polycyclic aromatic hydrocarbons and carbon in interplanetary dust particles and meteorites. Science 237, 56-59.

Allamandola, L.J., Sandford, S.A., Valero, G.J., 1988. Photochemical and thermal evolution of interstellar/precometary ice analogs. Icarus 76, 225-252.

Allamandola, L.J., Tielens, A.G.G.M., Barker, J.R., 1989. Interstellar polycyclic aromatic hydrocarbons: the infrared emission bands, the excitation/emission mechanism, and the astrophysical implications. Astrophys. J. Suppl. Ser. 71, 733-755.

Allamandola, L.J., Hudgins, D.M., Sandford, S.A., 1999. Modeling the Unidentified Infrared Emission with Combinations of Polycyclic Aromatic Hydrocarbons. Astrophys. J. 511, L115-L119.

Bernstein, M.P., Sandford, S.A., Allamandola, L.J., Chang, S., 1994. Infrared Spectrum of Matrix-Isolated Hexamethylenetetramine in $\mathrm{Ar}$ and $\mathrm{H}_{2} \mathrm{O}$ at Cryogenic Temperatures. J. Phys. Chem. 98, 12206-12210.

Bernstein, M.P., Sandford, S.A., Allamandola, L.J., Chang, S., Scharberg, M.A., 1995. Organic Compounds Produced by Photolysis of Realistic Interstellar and Cometary Ice Analogs Containing Methanol. Astrophys. J. 454, 327-344.

Bernstein, M.P., Sandford, S.A., Allamandola, L.J., 1996. Hydrogenated Polycyclic Aromatic

Hydrocarbons ( $\mathrm{H}_{\mathrm{n}}$-PAHs) and the 2940 and $2850 \mathrm{Wavenumber}$ (3.40 and 3.51 Micron) Infrared Emission Features. Astrophys. J. 472, L127-L130.

Bernstein, M.P., Sandford, S.A., Allamandola, L.J., Gillette, J.S., Clemett, S J., Zare, R.N., 1999.

UV Irradiation of Polycyclic Aromatic Hydrocarbons in Ices: Production of Alcohols, Quinones, and Ethers. Science 283, 1135-1138. 
Bernstein, M.P., Sandforl, S.A., Allamandola, L.J., 2000. H, C, N, and O Isotopic Substitution Studies of the 2165 Wiavenumbers ( 4.62 micron) "XCN" Feature Produced by UV Photolysis of Mixed Molecular Ices. Astrophys. J. 542, 894-897.

Bernstein, M.P., Dworkin, J.P., Sandford, S.A., Allamandola, L.J., 2001. Ultraviolet Irradiation of Naphthalene in $\mathrm{H}_{2} \mathrm{O}$ Ice: Implications for Meteorites and Biogenesis. Meteoritics and Planetary Science 36, 351-358.

Bregman, J.D., Hayward, T.L., Sloan, G.C., 2000. Discovery of the 11.2 micron polycylic aromatic hydrocarbon band in absorption toward Monoceros R2 IRS 3. Astrophys. J. 544, L75-L78.

Brooke, T.Y., Sellgren, K., Smith, R.G., 1996. A study of absorption features in the 3 micron spectra of molecular cloud sources with $\mathrm{H}_{2} \mathrm{O}$ ice bands. Astrophys. J. 459, 209-215.

Brooke, T.Y., Sellgren, K., Geballe, T.R., 1999. New 3 micron spectra of young stellar objects with $\mathrm{H}_{2} \mathrm{O}$ ice bands. Astrophys. J. 517, 883-900.

Brown, P.D., Charnley, S.B., 1990. Chemical models of interstellar gas-grain processes - I. Modelling and the effect of accretion on gas abundances and mantle composition in dense clouds. Mon. Not. R. astr. Soc. 244, 432-443.

Charnley, S.B., Tielens, A.G.G.M., Rodgers, S.D., 1997. Deuterated methanol in the Orion Compact Ridge. Astrophys. J. 482, L203-L206.

Chiar, J.E., Tielens, A.G.C.M., Whittet, D.C.B., Schutte, W.A., Boogert, A.C.A., Lutz, D., van Dishoeck, E.F., Bernstein, M.P., 2000. The compasition and distribution of dust along the line of sight towards the Gilactic Center. Astrophysical J. 537, 749-762.

Clemett, S.J., Maechling, C.R., Zare, R.N., Swan, P.D., Walker, R.M., 1993. Identification of Complex Aromatic Molecules in Individual Interplanetary Dust Particles. Science 262, 721 725.

Cody, III G.D., Alexander C.M.O.D., Tera, F., 2001. Solid state nuclear magnetic resonance studies of the Murchison organic macromolecule. Lunar Planet. Sci. Conf. 32, 2019.

Cronin, J.R., Pizzarello, S., Frye, J.S., 1987. ${ }^{13} \mathrm{C}$ NMR spectroscopy of the insoluble carbon of carbonaceous chondritis. Geochim. Cosmochim. Acta 51, 299-303.

Cronin, J.R., Chang, S., 1993. Organic matter in meteorites: Molecular and isotopic analyses of the Murchison meteorie, in: Greenberg, J.M., Mendoza-Gómez, C.X.. Pirronello, V. (Eds.). The Chemistry of Life'; Origins. Kluwer Academic Publishers, pp. 209-258. 
Dalgarno, A., Lepp, S., 1984. Deuterium fractionation mechanisms in interstellar clouds. Astrophys. J. 287, LA7-L50.

De Vries, M.S., Reihs, K., Wendt, H.R., Golden, W.G., Hunziker, H.E., Fleming, R., Peterson, E., Chang, S., 1993. A search for $\mathrm{C}_{60}$ in carbonaceous chondrites. Geochim. Cosmochim. Acta 57, 933-938.

d'Hendecourt, L.B., Allamandola, L.J., Greenberg, J.M., 1985. Time dependent chemistry in dense molecular clouds I. Grain surface reactions, gas/grain interactions and infrared spectroscopy. Astron. Astrophys. 152, 130-150.

Dworkin, J.P., Deamer, D.W., Sandford, S.A., Allamandola, L.J., 2001. Self-Assembling Amphiphilic Molecules: Synthesis in Simulated Interstellar/Precometary Ices. Proc. Nat. Acad. Sci. USA 98, 815-819.

Epstein, S., Krishnamurthy, R.V., Cronin, J.R., Pizzarello, S., Yuen, G.U., 1987. Unusual stable isotope ratios in amino acid and carboxylic acid extracts from the Murchison meteorite. Nature $326,477-479$.

Gardinier, A., Derenne, S., Robert, F., Behar, F., Largeau, C., Maquet, J., 2000. Solid state CP/MAS ${ }^{13} \mathrm{C}$ NMR of the insoluble organic matter of the Orgueil and Murchsion meteorites: Quantitative study. Earth Planet. Sci. Lett. 184, 9-21.

Geiss, J., Reeves, H., 1981. Deuterium in the Solar System. Astron. Astrophys. 93, 189-199. Hasegawa, T.I., Herbst, E., Leung, C.M., 1992. Models of gas-grain chemistry in dense interstellar clouds with complex organic molecules. Astrophys. J. Suppl. Ser. 82, 167-195.

Herbst, E., 1987. Gas phase chemical processes in molecular clouds, in: Hollenbach, D.J., Thronson, Jr., H.A. (Eds.), Interstellar Processes. D. Reidel, Dordrecht, pp. 611-629. Hollis, J.M., Snyder, L.E., Lovas, F.J., Buhl, D., 1976. Radio detection of interstellar DCO+. Astrophys. J. 209, L83-L85.

Hudgins, D.M., Sandford, S.A., Allamandola, L.J., 1994. Infrared Spectroscopy of Polycyclic Aromatic Hydrocarbon Cations I: Matrix-Isolated Naphthalene and Perdeuterated Naphthalene. J. Phys. Chem. 98, 4243-4253.

Jefferts, K.B., Penzias, A.A., Wilson, R.W., 1973. Deuterium in the Orion nebula. Astrophys. J. 179, L57-L59. 
Keller, L.P., Messenger, S., Flynn, G.J., Jacobsen, C., Wirick, S., 2000. Chemical and petrographic studies of molecular cloud materials preserved in interplanetary dust. Meteoritics and Planetary Science 35, A86-A87.

Kerridge, J.F., Chang, S., 1985. Survival of interstellar matter in meteorites: Evidence from carbonaceous material, in: Black, D.C., Matthews, M.S. (Eds.), Protostars and Planets II. University of Arizona Press, pp. 738-754.

Kerridge, J.F., Chang, S., Shipp, R., 1987. Isotopic characterization of kerogen-like material in the Murchison carbonaceous chondrite. Geochim. Cosmochim. Acta 51, 2527-2540.

Krishnamurthy, R.V., Epstein, S., Cronin, J.R., Pizzarello, S., Yuen, G.U., 1992. Isotopic and molecular analyses of hydrocarbons and monocarboxylic acids of the Murchison meteorite. Geochim. Cosmochim. Acta 56, 4045-4058.

Mauersberger, R., Henkel, C., Jacq, T., Walmsley, C.M., 1988. Deuterated methanol in Orion. Astron. Astrophys. 194, L1-L4.

Mauersberger, R., Wilson. T.L., Mezger, P.G., Gaume, R., Johnston, K.J., 1992. The internal structure of molecular clouds III. Evidence for molecular depletion in the NGC 2024 condensations. Astron. Astrophys. 256, 640-651.

McKeegan, K.D., Walker, R.M., Zinner, E., 1985. Ion Microprobe Isotopic Measurements of Individual Interplanetary Dust Particles. Geochim. Cosmochim. Acta 49, 1971-1987.

Messenger, S., 2000. Identification of molecular-cloud material in interplanetary dust particles. Nature 404, 968-971.

Millar, T.J., Bennett, A., Herbst, E., 1989. Deuterium fractionation in dense interstellar clouds. Astrophys. J. 340, 906-920.

Millar, T.J., Roberts, H., Markwick, A.J., Charnley, S.B., 2000. The role of $\mathrm{H}_{2} \mathrm{D}^{+}$in the deuteration of interstellar molecules. Phil Trans. R. Soc. Lond. A 358, 2535-2547.

Moore, M.H., Donn, B., Khanna, R., A'Hearn, M.F,. 1983. Studies of proton-irradiated cometary-type ice mixtures. Icarus 54, 388-405.

Norman, C., Silk, J., 1980. Clumpy Molecular Clouds: A Dynamic Model Self-Consistently Regulated by T Tauri Star Formation. Astrophys. J. 238, 158-174.

Pendleton, Y.J., Tielens, A.G.G.M., Tokunaga, A.T., Bernstein, M.P., 1999. The interstellar 4.62 micron band. Astrophys. J. 513, 294-304. 
Pizzarello, S., Krishnamurthy, R.V., Epstein, S., Cronin, J.R., 1991. Isotopic analyses of amino acids from the Murchison meteorite. Geochim. Cosmochim. Acta 55, 905-910.

Prasad, S.S., Tarafdar, S.P., 1983. UV Radiation Field Inside Dense Clouds: Its Possible Existance and Chemical Implications. Astrophys. J. 267, 603-609.

Puget, J.L., Leger, A., 1989. A new component of the interstellar matter: Small grains and large aromatic molecules. Annu. Rev. Astron. Astrophys. 27, 161-198.

Robert, F., Epstein S., 1982. The concentration and isotopic composition of hydrogen, carbon and nitrogen in carbonaceous meteorites. Geochim. Cosmochim. Acta 46, 81-95.

Robert, F., Gautier, D., Dubrulle, B., 2000. The Solar System D/H.ratio: Observations and theories. Space Science Reviews 92, 201-224.

Roelfsema, P.R., Cox, P., Tielens, A.G.G.M., Allamandola, L.J., Baluteau, J.-P., Barlow, M.J., Beintema, D., Boxhoorn, D.R., Cassinelli, J.P., Caux, E., Churchwell, E., Clegg, P. E., de Graauw, Th., Heras, A.M., Huygen, R., van der Hucht, K.A., Hudgins, D.M., Kessler, M.F., Lim, T., Sandford, S.A., 1996. SWS Observation of IR Emission Features Towards Compact HII Regions. Astron. Astrophys. 315, L289-L292.

Sandford, S.A., 1996. The Inventory of Interstellar Materials Available for the Formation of the Solar System. Meteoritics and Planetary Science 31, 449-476.

Sandford, S.A., Allamandola, L.J., 1993. Condensation and Vaporization Studies of $\mathrm{CH}_{3} \mathrm{OH}$ and $\mathrm{NH}_{3}$ Ices: Major Implications for Astrochemistry. Astrophys. J. 417, 815-825.

Sandford, S.A., Bernstein, M.P., Allamandola, L.J., Gillette, J.S., Zare, R.N., 2000. Deuterium enrichment of polycyclic aromatic hydrocarbons by photochemically induced exchange with eeuterium-rich cosmic ices. Astrophys. J. 538, 691-697.

Sandford, S.A., Bernstein, M.P., Dworkin, J.P., 2001. Assessment of the interstellar processes leading to deuterium enrichment in meteoritic organics. Meteoritics and Planetary Science $36,1117-1133$.

Sellgren, K., Brooke, T.Y., Smith, R.G., Geballe, T.R., 1995. A new 3.25 micron absorption feature towards Mon R2/IRS-3. Astrophys. J. 449, L69-L72.

Tegler, S.C., Weintraub, D.A., Allamandola, L.J., Sandford, S.A., Rettig, T.W., Campins, H., 1993. Detection of the 2165 inverse centimeters (4.619 micron) XCN Band in the Spectrum of L1551 IRS 5. Astrophys. J. 411, 260-265. 
Teixeira, T.C., Devlin, J.P., Buch, V. Emerson, J.P., 1999. Discovery of solid HDO in grain mantles. Astron. Astrophys. 347, L19-L22.

Tielens, A.G.G.M., 1983. Surface chemistry of deuterated molecules. Astron. Astrophys. 119, 177-184.

Tielens, A.G.G.M., 1992. The D/H ratio in molecular clouds, in: Singh, P.D. (Ed.), Astrochemistry of Cosmic Phenomena. Kluwer, Dordrecht, pp. 91-95.

Tielens, A.G.G.M., 1997. Deuterium and interstellar chemical processes, in: Bernatowicz, T.J., Zinner, E. (Eds.), Astrophysical Implications of the Laboratory Study of Presolar Materials. Amer. Inst. of Physics, pp. 523-544.

Tielens, A.G.G.M., Hagen, W., 1982. Model calculations of the molecular composition of interstellar grain mantles. Astron. Astrophys. 114, 245-260.

Whittet, D.C.B., Schutte, W.A., Tielens, A.G.G.M., Boogert, A.C.A., de Graauw, Th., Ehrenfreund, P., Gerakines, P.A., Helmich, F.P., Prusti, T., van Dishoeck, E.F., 1996. An ISO SWS view of interstellar ices - First results. Astron. Astrophys. 315, L357-L360.

Yang, J., Epstein, S., 1983. Interstellar organic matter in meteorites. Geochim. Cosmochim. Acta 47, 2199-2216.

Zinner, E., 1988. Interstellar cloud material in meteorites, in: Kerridge, J.F., Matthews, M.S. (Eds.), Meteorites and the Early Solar System. University of Arizona Press, pp. 956-983. Zinner, E., 1997. Presolar material in meteorites: An overview, in: Bernatowicz, T.J., Zinner, E. (Eds.), Astrophysical Implications of the Laboratory Study of Presolar Materials. Amer. Inst. Priys. Press, pp. 3-26. 


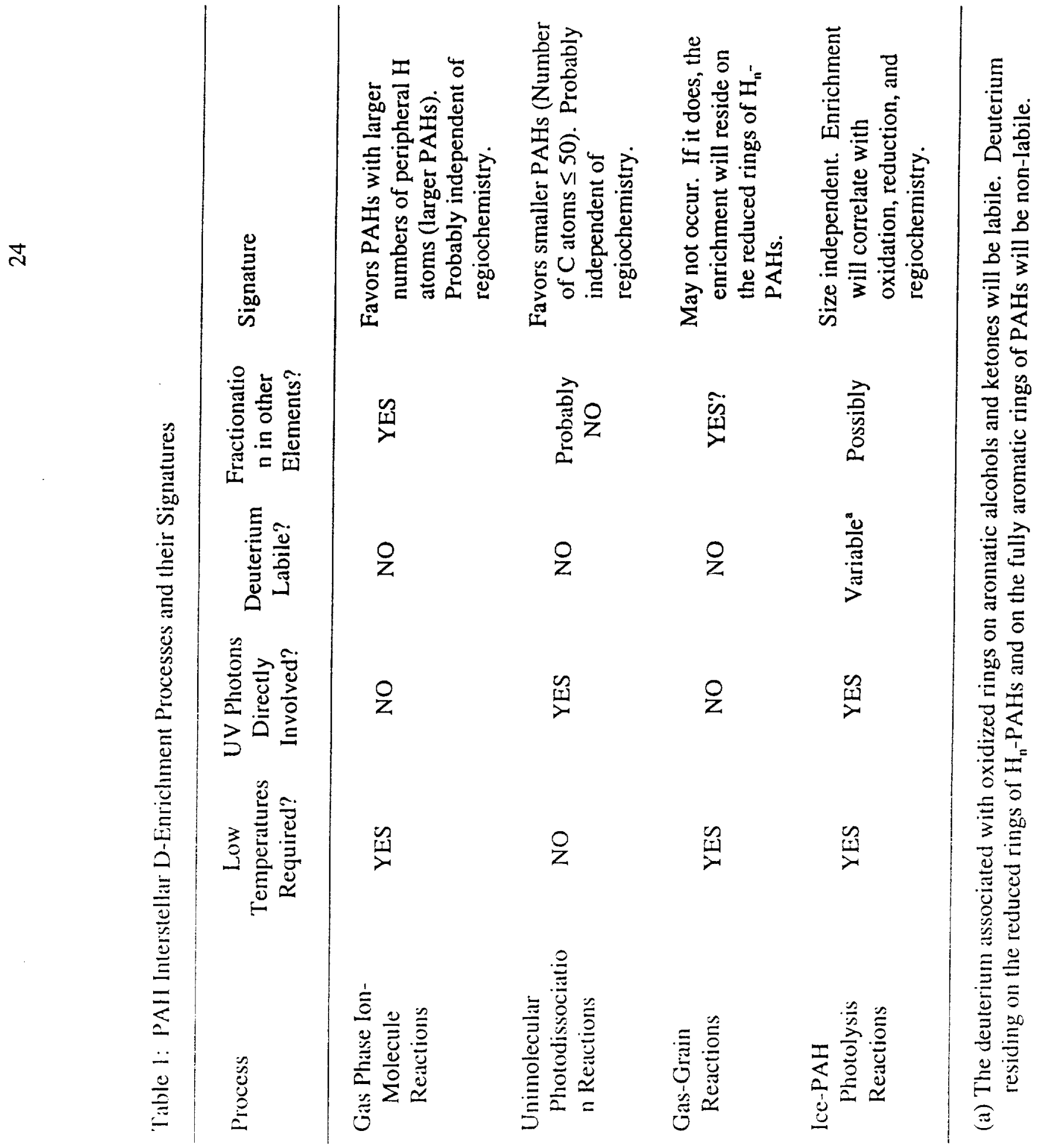




\section{FIGURE CAPTIONS}

Figure 1 - PAHs become enriched in D via gas phase ion-molecule reactions in a two step process (shown here for the PAH pyrene). First, a PAH reacts with $\mathrm{H}_{2} \mathrm{D}^{+}$to pick up an excess $\mathrm{D}$ atom and charge. Subsequent reaction with an $\mathrm{e}^{-}$neutralizes the charge and the PAH eliminates an excess $\mathrm{H}$ or $\mathrm{D}$ atom. This process should initially preferentially deuterate larger PAHs in dense clouds, but all gas phase PAHs would ultimately become $D$ enriched as they approach steady-state equilibrium with the gas phase.

Figure 2 - In dense clouds where $\mathrm{H} / \mathrm{H}_{2}$ is large, gas-grain reactions primarily serve to hydrogenate, and D-enrich, species in the ice. It is not clear if PAHs can be hydrogenated in this manner, but to the extent that this process occurs, any $D$ enrichments generated would be expected to correlate with the presence of $H_{n}-P A H s$ and cyclic aliphatics.

Figure 3 - Gas phase PAHs can become D-enriched by repeated unimolecular photodissociation events that favor retention of $D$ over $H$. The extent of the enrichment depends on the size of the PAH, with the most significant fractionations occurring for PAHs in the range of sizes spanned by naphthalene $\left(\mathrm{C}_{10} \mathrm{H}_{8}\right)$ and hexabenzocoronene $\left(\mathrm{C}_{42} \mathrm{H}_{18}\right)$. Enrichment in larger PAHs is not expected because they have large numbers of vibrational modes and are stable against photolytic bond rupture. D enrichment by unimolecular photodissociation does not require low ambient temperatures.

Figure 4- The UV photolysis of PAHs in D-enriched ices results in rapid D enrichment of the PAHs through (a) aromatic $\mathrm{D} \rightarrow \mathrm{H}$ exchange, (b) D-atom addition, and (c) exchange through keto-enol tautomerism. Deuterium enrichments produced in this manner are largely independent of PAH molecular size, but should show specific regiochemical patterns and would be expected to correlate with the presence of both reduced PAHs $\left(\mathrm{H}_{\mathrm{n}}\right.$-PAHs) and oxidized PAHs (aromatic alcohols, ketones, and ethers). 
Figure 5 - Substitution of $\mathrm{D}$ for $\mathrm{H}$ moves the (a) $\mathrm{C}-\mathrm{H}$ stretching and (b) $\mathrm{C}-\mathrm{H}$ bending mode vibrations of organics (represented here by several different PAH species) to longer wavelengths. The $\mathrm{C}-\mathrm{D}$ stretching and bending mode vibrations fall in wavelength regions (shaded gray) that are blocked from ground-based and airborne observation by telluric $\mathrm{CO}_{2}$.

Figure 6 - The infrared spectrum of a mixture of PAHs that contain D-enriched members. The $\mathrm{D} / \mathrm{H}$ ratio of the total mixture is 0.05 . The gray bands represent spectral regions that are blocked from ground-based observation by telluric $\mathrm{H}_{2} \mathrm{O}$ and $\mathrm{CO}_{2}$. 
Figure 1
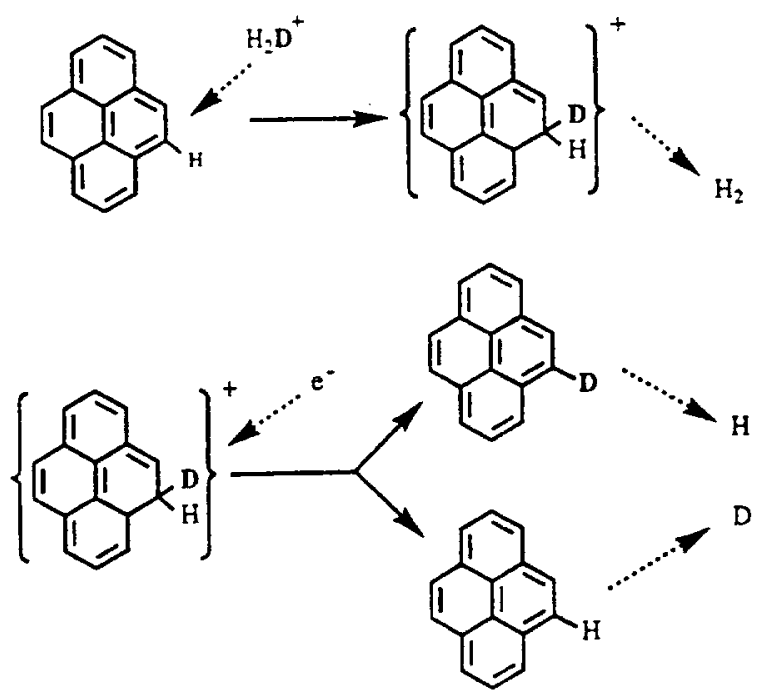

Figure 2

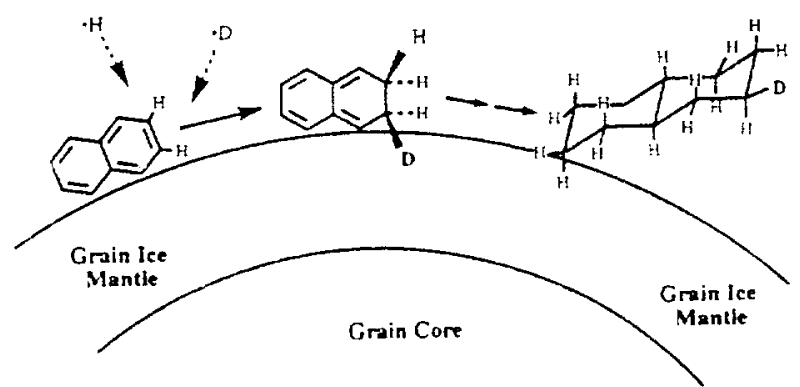


Figure 3

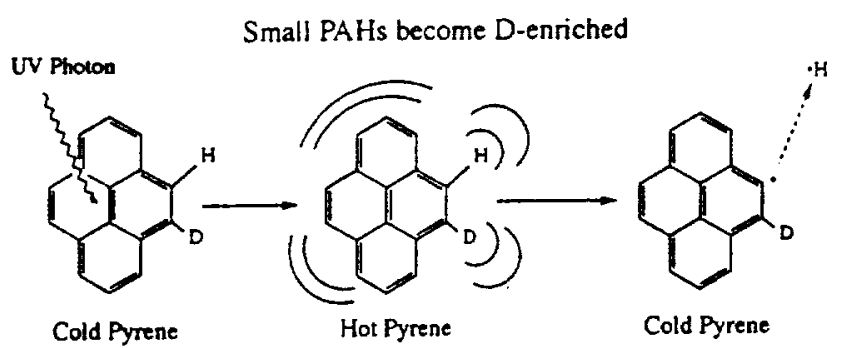

Larger PAHs Don't

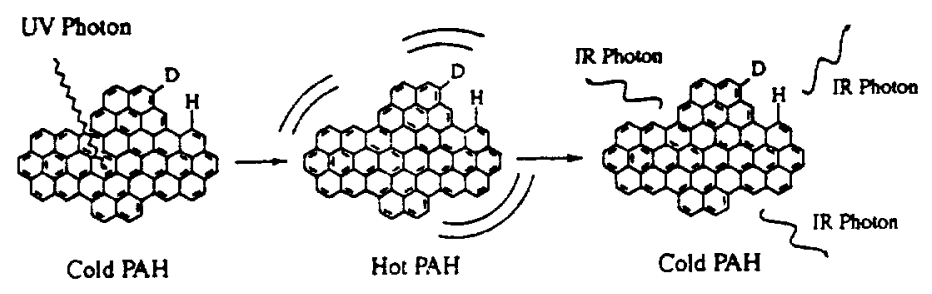

Figure 4

(a)

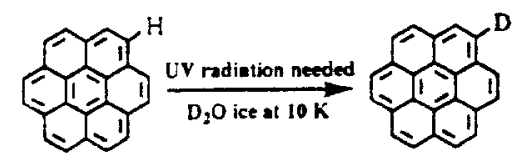

(b)

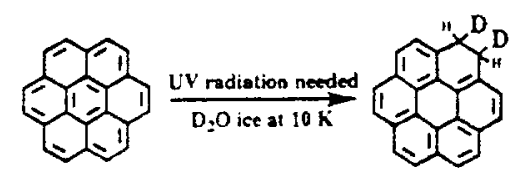

(c)

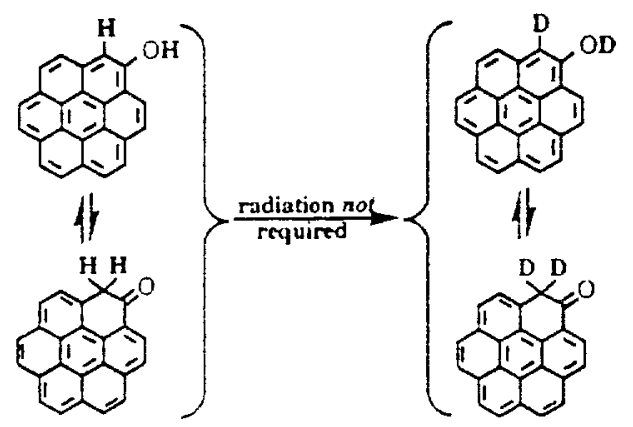


Figure 5a

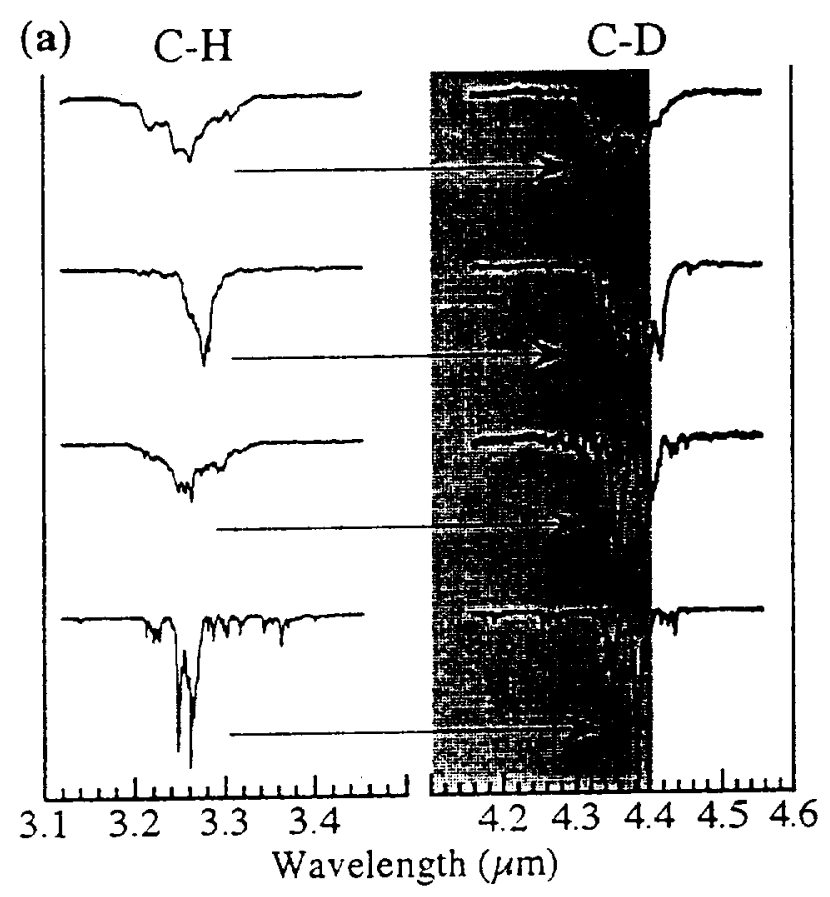

Figure 5b

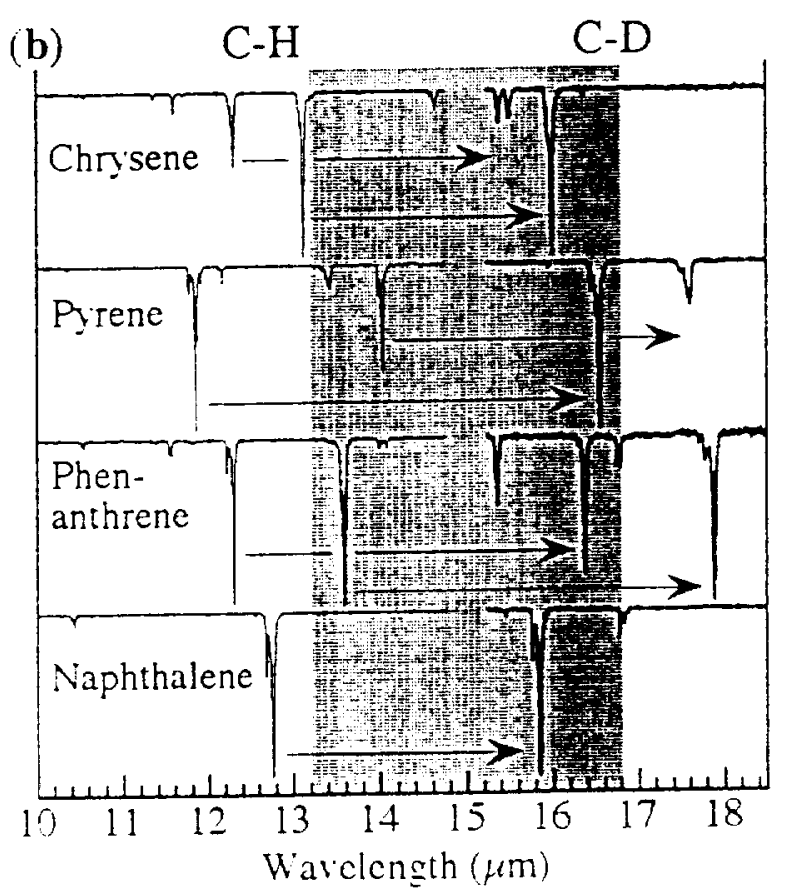


Figure 6

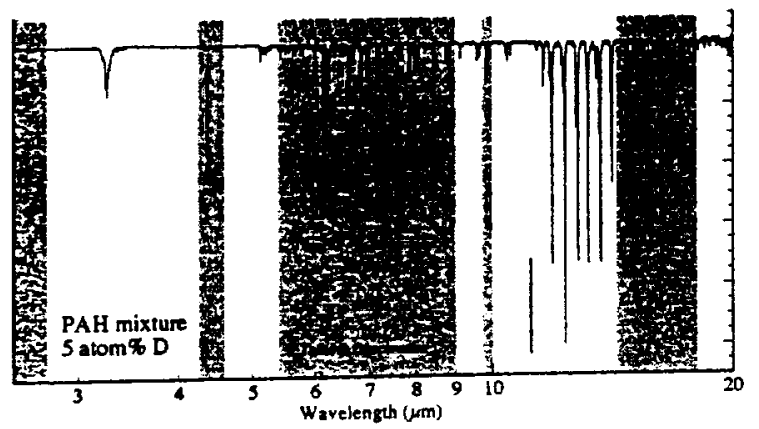

